\title{
Spectrum of qubit oscillations from Bloch equations
}

\author{
Rusko Ruskov* and Alexander N. Korotkov ${ }^{\dagger}$ \\ Department of Electrical Engineering, University of California, Riverside, CA 92521.
}

(October 24, 2018)

We have developed a formalism suitable for calculation of the output spectrum of a detector continuously measuring quantum coherent oscillations in a solid-state qubit, starting from microscopic Bloch equations. The results coincide with that obtained using Bayesian and master equation approaches. The previous results are generalized to the cases of arbitrary detector response and finite detector temperature.

\section{INTRODUCTION}

Quantum coherent (Rabi) oscillations in a two-level system represent a simple and fundamental example of a nontrivial quantum behavior. 1 Recently increased interest in this subject is obviously related to the use of twolevel systems (qubits) as building blocks of a prospective quantum computer. The emphasis has naturally shifted from traditional observations of Rabi oscillations in ensembles of two-level atoms to the studies of single qubits. Concentrating in this paper on solid-state qubits, let us mention recent demonstrations of singlequbit quantum coherent oscillations in both time 3 and frequency 5 domains. (Rabi oscillations in individual quantum dots have been also demonstrated by traditional optical means; 6 however, we will discuss only solid-state qubits with electronic readout.)

Even though experiments of Refs. 35 have been done with single qubits, their results are essentially ensembleaveraged, and as a consequence, the problem of a quantum state collapse due to measurement is not very important. Another possible experimental setup (for which the collapse is of the major importance) is a continuous monitoring of single-qubit quantum oscillations (Fio. 1). Such setup is similar to that used in experiments with the difference that the detector output signal $I(t)$ was actually not studied experimentally.

The basic questions in prospective experiments of the type shown in Fig. 1 are the following: 1) what is the effect of continuous quantum measurement on the qubit evolution, 2) how the detector output $I(t)$ looks like, and 3 ) what is the relation between detector output and qubit evolution? Some answers to these questions have been obtained recently (see, e.g., Ref 9 and references therein); however, the subject is still active and quite controversial. (The main reason of controversy is the necessity to go beyond the Schrödinger equation to describe continuous collapse due to measurement.)

In this paper we will consider a relatively simple ques-

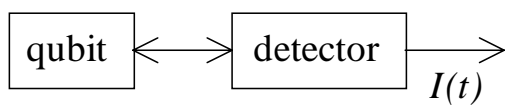

FIG. 1. Schematic of a single solid-state qubit continuously measured by a detector.

tion: what is the spectral density $S_{I}(\omega)$ of the detector output $I(t)$ and how high is the spectral peak corresponding to quantum oscillations of the qubit state? (Notice that we assume detector output $I(t)$ to be a classical magnitude, so $S_{I}(\omega)$ does not depend on a particular method of further signal processing.) This question has been addressed already in a number of papers (see, e.g., Refs.10 18) using various techniques.

Spectral density $S_{(}(\omega)$ has been calculated using Bayesian formalism, 19 in Ref 10 for the case of a weakly responding (linear) detector. In particular, it has been shown that the spectral peak $S_{I}(\Omega)$ at the frequency $\Omega$ of quantum oscillations cannot be higher than $4 S_{0}$ where $S_{0}$ is the noise pedestal due to intrinsic detector noise. It has been also shown that the results of the Bayesian formalism for $S_{I}(\omega)$ exactly coincide with the result, 10,11 obtained using the standard master equation formalism. In spite of the same results, the interpretations are quite different since the Bayesian formalism describes individual monitoring of quantum evolution in time and treats $I(t)$ as a classical measurement result, while the master equation formalism can describe only ensemble-averaged magnitudes and in some sense should treat $I(t)$ as a quantum operator. (The results of two formalisms coincide because $S_{I}(\omega)$ is essentially an average quantity. In more general situlations, for example, for a quantum feedback analysis 20 the results are not the same since the master equation formalism fails.)

The results for $S_{I}(\omega)$ have been confirmed in Ref.12 using a somewhat different approach based on the general theory of linear detectors. In Ref. 13 the results have been confirmed using the approach of quantum trajectories 21.22 adopted from quantum optics (this approach is similar to the Bayesian formalism). It has been also shown 13 that the same formulas for $S_{I}(\omega)$ remain valid even when the condition of weakly responding detector is not satisfied (the detector response $\Delta I$ to the change of the qubit state is comparable to the average current $\left.I_{0}\right)$.

A different result for $S_{I}(\omega)$ has been obtained in Ref.14 (the studied system is slightly different from the type shown in Fig. If however, it is essentially similar). In particular, the calculated ratio $\left[S_{I}(\Omega)-S_{0}\right] / S_{0}$ can be 
arbitrary large (not limited by 4). In our opinion, this is because the result of Ref. 14 includes the contribution from zero-point oscillations which are not measurable in a straightforward way. In principle, zero-point oscillations can be sensed in an experiment; however, it would necessarily require quantum (non-classical) interaction between the detector [which outputs $I(t)$ ] and the next stage. In other words, it would require measurement of $S_{I}(\omega)$ without ever measuring $I(t)$. A simple example of such setup is the absorption/emission of photons at resonant frequency $\Omega$. A more sophisticated example of such measurement of qubit oscillations has been considered in Ref.15. The idea is to use rotating measurement basis, in which there are essentially no oscillations, but rather jumps between two stationary states due to external noise. Formally shifting the zero-frequency spectrum of such continuous measurement to the frequency $\Omega$, one can obtain arbitrary high spectral peak. (There is no restriction on the ratio $S_{I}(\omega=0) / S_{0}$ in a strong dephasing case.10 13) Such setups, however, are not the subject of the present paper since we limit ourselves to the straightforward case of Fig. 11 with $I(t)$ being usual classical signal which can be amplified further by any good amplifier. Also, we do not consider here the quantum feedback setups, which can provide arbitrary high spectral peak of the detector current at the oscillation frequency $\Omega .20$

The main result $\left[S_{I}(\Omega)-S_{0}\right] / S_{0} \leq 4$ for a simple setum seems to contradict the experimental results of Ref.23 which claim the measurement of $S_{I}(\omega)$ from a single spin precession in an STM-based setup (a significantly different, but still an analogous experiment). In a similar recent experiment 24 the maximum observed peak-topedestal ratio for a measurement of a single spin precession was a little less than 4.25 We cannot explain the disagreement between the theory and experiment of Ref.23. however, we note that in the recent theoretical paper 16 which considers a somewhat similar setup, the possibility of a relatively high spectral peak has not been confirmed. The spectral peak of $S_{I}(\omega)$ due to qubitgscillations has been also considered theoretically in Ref 17 ; however, the peak magnitude has not been calculated.

Let us finally mention one more theoretical approach (developed by S. A. Gurvitz) to the analysis of detector output $I(t)$ based on Bloch equations which describe the evqution of the coupled "qubit+detector" density matrix.26 The advantage of this approach is the straightforward microscopic derivation of Bloch equations from the Schrödinger equation, while in the approaches mentioned above (Bayesian, master equation, quantum trajectory) the collapse ansatz is either explicitly or implicitly used. The Bloch equation approach has been used to calculate some statistical characteristics of $I(t)$ in Ref.18; however, the spectral density $S_{I}(\omega)$ has been obtained only at the frequencies lower than the ensemble dephasing rate and so the spectral peak due to quantum oscillations has been out of scope of Ref 18 .

In this paper we show how the Bloch equations can be used to calculate the detector output spectral density

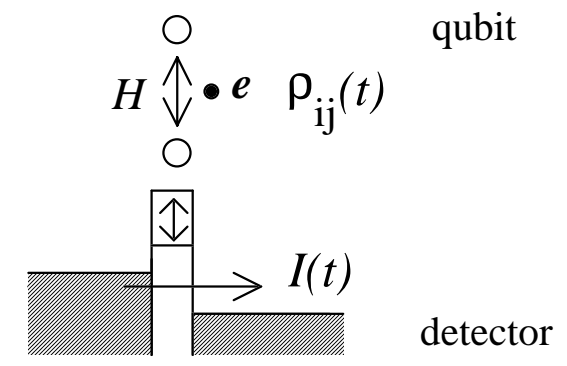

FIG. 2. Small transparency QPC (tunnel junction) as a qubit state detector. The barrier height depends on the electron position in the double-dot system.

$S_{I}(\omega)$ for a particular measurement setup (Fig. 2). The case of an arbitrary qubit coupling with detector and finite detector temperature is considered. We prove that the results for $S_{I}(\omega)$ coincide with that obtained previously (in a narrower validity range) by the master equation and Bayesian approaches. The Bayesian results are generalized to the case of arbitrary response factor and finite detector temperature; it is shown that the equivalence of results of the three approaches still holds in this case.

\section{THE SYSTEM AND BLOCH EQUATIONS}

We consider the system (Fig. 2) intregued in-Ref.26 and studied extensively after that.6 $43.17,29,20.22$ The qubit is represented by a single electron in a double quantum dot. The detector is a quantum point contact (QPC) whose barrier height depends on the electron position, so the current $I$ through QPC measures the qubit state in the basis of localized states $|1\rangle$ and $|2\rangle$. We will limit ourselves by the case of small transparency QPC which is equivalent to a simple tunnel junction. The Hamiltonian of the system,

$$
\mathcal{H}=\mathcal{H}_{Q B}+\mathcal{H}_{D E T}+\mathcal{H}_{I N T},
$$

describes the qubit, detector, and their interaction:

$$
\begin{aligned}
\mathcal{H}_{Q B}= & \frac{\varepsilon}{2}\left(c_{1}^{\dagger} c_{1}-c_{2}^{\dagger} c_{2}\right)+H\left(c_{1}^{\dagger} c_{2}+c_{2}^{\dagger} c_{1}\right), \\
\mathcal{H}_{D E T}= & \sum_{l} E_{l} a_{l}^{\dagger} a_{l}+\sum_{r} E_{r} a_{r}^{\dagger} a_{r} \\
& +\sum_{l, r} M a_{l}^{\dagger} a_{r}+\text { H.c. }, \\
\mathcal{H}_{I N T}= & \sum_{l, r} \frac{\Delta M}{2}\left(c_{1}^{\dagger} c_{1}-c_{2}^{\dagger} c_{2}\right) a_{l}^{\dagger} a_{r}+\text { H.c. }
\end{aligned}
$$

(for simplicity $M$ and $\Delta M$ are assumed to be real and energy-independent). The average detector currents corresponding to the qubit states $|1\rangle$ and $|2\rangle$ are equal to $I_{1}=2 \pi(M+\Delta M / 2)^{2} \rho_{l} \rho_{r} e^{2} V / \hbar$ and $I_{2}=2 \pi(M-$ $\Delta M / 2)^{2} \rho_{l} \rho_{r} e^{2} V / \hbar$, correspondingly ( $V$ is the voltage 
across the tunnel junction, $e$ is the electron charge, and $\rho_{l, r}$ are the densities of states in the electrodes), while the corresponding detector noises have white spectrum and are given by the Schottky formula:

$$
S_{1,2}=2 e I_{1,2} \text {. }
$$

Note that the detector voltage $V$ is assumed to be large enough, so that the typical quantum noise frequency $e V / \hbar$ is much higher than all relevant frequencies.27

In the following we will distinguish the weaklyresponding limit, $|\Delta I| \ll I_{0}$, where $\Delta I \equiv I_{1}-I_{2}$ is the detector response and $I_{0} \equiv\left(I_{1}+I_{2}\right) / 2$, and the finite response case, $|\Delta I| \sim I_{0}$. Notice that the word "coupling" is reserved for a different combination of parameters: $\mathcal{C} \equiv \hbar(\Delta I)^{2} / S_{0} H$ [here $S_{0} \equiv\left(S_{1}+S_{2}\right) / 2$ ], which affects the quality factor of quantum oscillations of the qubit. The frequency of unperturbed oscillations (without detector) is equal to $\Omega \equiv \sqrt{4 H^{2}+\varepsilon^{2}} / \hbar$, where $H$ is the qubit tunneling matrix element (assumed to be real) and $\varepsilon$ is the qubit energy asymmetry.

Our starting point is the Bloch equations 26 describing the ensemble averaged evolution of the density matrix $\rho_{i j}^{n}$ in which the subscripts $(i, j=1,2)$ label the qubit state while $n$ is the number of electrons passed through the detector (only diagonal in $n$ matrix elements are considered because the nondiagonal elements decay very fast) For our system the Bloch equations are the following 26 , 9

$$
\begin{aligned}
\dot{\rho}_{11}^{n}= & -\frac{I_{1}^{+}+I_{1}^{-}}{e} \rho_{11}^{n}+\frac{I_{1}^{+}}{e} \rho_{11}^{n-1}+\frac{I_{1}^{-}}{e} \rho_{11}^{n+1} \\
& -2 \frac{H}{\hbar} \operatorname{Im} \rho_{12}^{n}, \\
\dot{\rho}_{22}^{n}= & -\frac{I_{2}^{+}+I_{2}^{-}}{e} \rho_{22}^{n}+\frac{I_{2}^{+}}{e} \rho_{22}^{n-1}+\frac{I_{2}^{-}}{e} \rho_{22}^{n+1} \\
& +2 \frac{H}{\hbar} \operatorname{Im} \rho_{12}^{n}, \\
\dot{\rho}_{12}^{n}= & -\frac{I_{1}^{+}+I_{1}^{-}+I_{2}^{+}+I_{2}^{-}}{2 e} \rho_{12}^{n}+\frac{\sqrt{I_{1}^{+} I_{2}^{+}}}{e} \rho_{12}^{n-1} \\
& +\frac{\sqrt{I_{1}^{-} I_{2}^{-}}}{e} \rho_{12}^{n+1}+\mathrm{i} \frac{\varepsilon}{\hbar} \rho_{12}^{n}+\mathrm{i} \frac{H}{\hbar}\left(\rho_{11}^{n}-\rho_{22}^{n}\right) .
\end{aligned}
$$

Here

$$
I_{i}^{+}=\frac{I_{i}}{1-\exp (-e V / T)}, I_{i}^{-}=I_{i}^{+} \exp (-e V / T)
$$

are the partial currents in two directions $\left(I_{i}=I_{i}^{+}-I_{i}^{-}\right)$ and $T$ is the detector electron temperature. Notice that this temperature is different from what was considered in Refs 1012 . In those papers the effect of nonzero temperature of a passive environment coupled to the qubit was studied, so the important parameter was $T / \hbar \Omega$. In this paper (similar to Ref22) we consider the effective detector temperature and the important parameter is $T / \mathrm{eV}$, while a finite temperature $T$ always implies $T \gg \hbar \Omega$. (The phonon temperature in the vicinity of the qubit may still be low, since $T$ is only the electron temperature.) The density matrix $\rho_{i j}^{n}$ obeys the natural normalization condition $\sum_{n}\left(\rho_{11}^{n}+\rho_{22}^{n}\right)=1$.

Notice that tracing Bloch equations (6)-(8) over the detector degree of freedom $n$, one obtains a conventional master equation for the qubit:

$$
\begin{aligned}
& \dot{\rho}_{11}=-2 \frac{H}{\hbar} \operatorname{Im} \rho_{12}, \quad \rho_{11}+\rho_{22}=1, \\
& \dot{\rho}_{12}=\mathrm{i} \frac{\varepsilon}{\hbar} \rho_{12}+\mathrm{i} \frac{H}{\hbar}\left(\rho_{11}-\rho_{22}\right)-\Gamma \rho_{12},
\end{aligned}
$$

where $\rho_{i j}=\sum_{m} \rho_{i j}^{n}$ and the ensemble decoherence rate $\Gamma$ is equal to 262

$$
\begin{aligned}
\Gamma= & \frac{\left(\sqrt{I_{1}^{+}}-\sqrt{I_{2}^{+}}\right)^{2}}{2 e}+\frac{\left(\sqrt{I_{1}^{-}}-\sqrt{I_{2}^{-}}\right)^{2}}{2 e} \\
= & \frac{\left(\sqrt{I_{1}}-\sqrt{I_{2}}\right)^{2}}{2 e} \operatorname{coth}(e V / 2 T) .
\end{aligned}
$$

(The decoherence rate $\gamma$ for a single system without ensemble averaging is different - see Section V.)

\section{SPECTRAL DENSITY VIA MACDONALD'S FORMULA}

The Bloch equations couple the qubit evolution and the number $n$ of electrons passed through the detector. So, to calculate the spectral density $S_{I}(\omega)$ of the detector current, we need to express $S_{I}(\omega)$ in terms of $n$. This can be easily done for the classical random process $I(t)$ using the MacDonald's formulates

$$
S_{I}(\omega)=2 \omega \int_{0}^{\infty} \frac{d\left\langle Q^{2}(\tau)\right\rangle}{d \tau} \sin (\omega \tau) d \tau,
$$

where $\left\langle Q^{2}(\tau)\right\rangle=\left\langle\left(\int_{t}^{t+\tau} I\left(t^{\prime}\right) d t^{\prime}-\langle I\rangle \tau\right)^{2}\right\rangle$ and averaging is over time $t$ (MacDonald's formula have been also used in Ref.16). In our case the average current is equal to $I_{0}$ (see below) for a non-zero qubit tunneling $H$, so

$$
\left\langle Q^{2}(\tau)\right\rangle=e^{2}\left\langle n^{2}(\tau)\right\rangle-\left(I_{0} \tau\right)^{2},
$$

where $\left\langle n^{2}(\tau)\right\rangle$ is the average square of the number of electrons passed through the detector during time interval $\tau$.

To calculate $\left\langle n^{2}(\tau)\right\rangle$ we can use the Bloch equations and the obvious relation

$$
\left\langle n^{2}(\tau)\right\rangle=\sum_{n} n^{2}\left[\rho_{11}^{n}(\tau)+\rho_{22}^{n}(\tau)\right]
$$

However, the situation is not too simple because the left hand side contains the averaging over time while the right hand side is essentially ensemble averaging which depends on the initial condition $\rho_{i j}^{n}(0)$. Quite naturally we should assume $n=0$ at $\tau=0$, so that $\rho_{i j}^{n}(0)=\delta_{n 0} \rho_{i j}(0)$, 
but the question about the choice of $\rho_{i j}(0)$ remains unclear because the qubit state actually oscillates in time (for a nonzero $H$, which case we always assume below). A natural choice is to use the stationary value: $\rho_{i j, s t}=\lim _{t \rightarrow \infty} \sum_{n}\left[\rho_{i j}^{n}(t)+\rho_{i j}^{n}(t)\right]$, and it is possible to prove that this choice is really correct in the following way.

As we know from the Bayesian formalism 9 we can monitor the oscillating evolution of the qubit density matrix $\rho_{i j}(t)$ in an individual realization of the experiment using the detector output $I(t)$. This at least means that $\rho_{i j}(t)$ exists (even though it cannot be obtained using Bloch equations because they imply ensemble averaging). So, the correct procedure of calculating $\left\langle n^{2}(\tau)\right\rangle$ would be the following. The right hand side of Eq. (15) should be calculated for various initial values $\rho_{i j}(\tau=0)$ corresponding to values $\rho_{i j}(t)$ in a sufficiently long realization of a process, and then the result should be averaged over the time $t$ [i.e. weighted proportionally to the occurrence frequency of various $\left.\rho_{i j}\right]$. Now it is very important that the Bloch equations (6)-(8) are linear in respect to the initial condition. This means that instead of averaging the result for $n^{2}(\tau)$ over initial condition $\rho_{i j}(\tau=0)$, we can use the initial condition which is itself the value averaged over time, i.e. stationary value $\rho_{i j, s t}$ discussed above (of course, we implicitly use the process ergodicity). This ends the proof.

Thus, to calculate $S_{I}(\omega)$ we should solve the Bloch equations starting from the stationary initial condition $\rho_{i j}^{n}(0)=\delta_{n 0} \rho_{i j, s t}$, then calculate $\left\langle n^{2}(\tau)\right\rangle$ using Eq. (15), and then use MacDonald's formula (13) to obtain $S_{I}(\omega)$. Notice that the stationary state $\rho_{i j, s t}$ can be easily obtained from the master equations $(10)-(11)$ and the condition $\dot{\rho}_{i j}=0$, that gives $($ at $H \neq 0$ )

$$
\rho_{11, s t}=\rho_{22, s t}=1 / 2, \quad \rho_{12, s t}=0 .
$$

(The stationary state would be different if the-qubit had an extra coupling to a passive environment,10.11 however, we do not consider such case.)

One can use this method to calculate $S_{I}(\omega)$ in a straightforward way (we have done it numerically); however, it is better to use an analytical simplification calculating directly $d\left\langle n^{2}(\tau)\right\rangle / d \tau=\sum_{n} n^{2}\left[\dot{\rho}_{11}^{n}(\tau)+\dot{\rho}_{22}^{n}(\tau)\right]$. Using Eqs. (6) and (7) and shifting summation over $n$ in terms containing $\rho_{i i}^{n}$, , one gets the equation

$$
\begin{aligned}
\frac{d\left\langle n^{2}(\tau)\right\rangle}{d \tau} & =\frac{I_{0}}{e}\left(2\langle n(\tau)\rangle+\operatorname{coth} \frac{e V}{2 T}\right) \\
& +\frac{\Delta I}{e}\left(\mathcal{A}(\tau)+\frac{z(\tau)}{2} \operatorname{coth} \frac{e V}{2 T}\right)
\end{aligned}
$$

where

$$
\begin{aligned}
& \mathcal{A}(\tau) \equiv \sum_{n} n\left[\rho_{11}^{n}(\tau)-\rho_{22}^{n}(\tau)\right] \\
& \langle n(\tau)\rangle \equiv \sum_{n} n\left[\rho_{11}^{n}(\tau)+\rho_{22}^{n}(\tau)\right]
\end{aligned}
$$

$$
z(\tau) \equiv \sum_{n}\left[\rho_{11}^{n}(\tau)-\rho_{22}^{n}(\tau)\right]
$$

Notice that $z(\tau)=0$ since the evolution starts from the stationary state, $\rho_{i j}^{n}(0)=\delta_{n 0} \rho_{i j, s t}$, so the corresponding term in Eq. (17) vanishes.

To calculate $\langle n(\tau)\rangle$, we again use Eqs. (6) and (7), shift summation over $n$, and obtain the equation

$$
d\langle n(\tau)\rangle / d \tau=I_{0}+z(\tau) \Delta I / 2 .
$$

Since the last term vanishes because of $z(\tau)=0$ and since $\langle n(0)\rangle=0$, we obtain a simple result $\langle n(\tau)\rangle=I_{0} \tau$. In particular, this means that the average detector current is equal to $I_{0}$ (we have used this result above).

One can see that the term $2 I_{0}\langle n(\tau)\rangle / e=2 I_{0}^{2} \tau$ from Eq. (17) exactly cancels the contribution from the derivative of the last term of Eq. (14). The term $\left(I_{0} / e\right) \operatorname{coth}(e V / 2 T)$ from Eq. (17) after being plugged into MacDonald's formula (13) gives the constant noise pedestal $2 e I_{0} \operatorname{coth}(e V / 2 T$ ) (as usual, we should use a smooth integral cutoff at high frequencies). In this way we get equations

$$
\begin{aligned}
S_{I}(\omega) & =S_{0} \operatorname{coth} \frac{e V}{2 T}+2 \omega e \Delta I \int_{0}^{\infty} \mathcal{A}(\tau) \sin (\omega \tau) d \tau \\
= & S_{0} \operatorname{coth} \frac{e V}{2 T}+2 e \Delta I \int_{0}^{\infty} \frac{d \mathcal{A}(\tau)}{d \tau} \cos (\omega \tau) d \tau
\end{aligned}
$$

which express $S_{I}(\omega)$ via $\mathcal{A}(\tau)$ or $\dot{\mathcal{A}}(\tau)$ [the last equation is obtained using integration by parts and taking into account $\mathcal{A}(0)=0$ ].

To calculate $\mathcal{A}(\tau)$ (or $\dot{\mathcal{A}}$ ), we notice that the Bloch equations (6)-(8) couple the dynamics of $\mathcal{A}(\tau)$ with two more magnitudes

$$
\begin{aligned}
& \mathcal{Y}(\tau) \equiv \sum_{n} n \operatorname{Im} \rho_{12}^{n}(\tau) \\
& \mathcal{X}(\tau) \equiv \sum_{n} n \operatorname{Re} \rho_{12}^{n}(\tau)
\end{aligned}
$$

via equations

$$
\begin{aligned}
\dot{\mathcal{A}} & =\frac{\Delta I}{2 e}-4 \frac{H}{\hbar} \mathcal{Y}+\frac{I_{0}}{e} z \\
\dot{\mathcal{Y}} & =\frac{H}{\hbar} \mathcal{A}+\frac{\varepsilon}{\hbar} \mathcal{X}-\Gamma \mathcal{Y}+b \operatorname{Im} \rho_{12}, \\
\dot{\mathcal{X}} & =-\frac{\varepsilon}{\hbar} \mathcal{Y}-\Gamma \mathcal{X}+b \operatorname{Re} \rho_{12},
\end{aligned}
$$

where $b=\left[\left(I_{1}^{+} I_{2}^{+}\right)^{1 / 2}-\left(I_{1}^{-} I_{2}^{-}\right)^{1 / 2}\right] / e$. Because of the stationary initial conditions $z(\tau)=\rho_{12}(\tau)=0$, so the equations are further simplified and become a closed system:

$$
\begin{aligned}
& \dot{\mathcal{A}}=(\Delta I / 2 e)-4(H / \hbar) \mathcal{Y} \\
& \dot{\mathcal{Y}}=(H / \hbar) \mathcal{A}+(\varepsilon / \hbar) \mathcal{X}-\Gamma \mathcal{Y} \\
& \dot{\mathcal{X}}=-(\varepsilon / \hbar) \mathcal{Y}-\Gamma \mathcal{X}
\end{aligned}
$$


Solving these equations with the initial condition $\mathcal{A}(0)=$ $\mathcal{X}(0)=\mathcal{Y}(0)=0$, one can obtain $\mathcal{A}(\tau)$ and therefore $S_{I}(\omega)$.

In the case of a symmetric qubit, $\varepsilon=0$, the evolution of $\mathcal{X}$ is decoupled and one can find the analytical solution

$$
\frac{d \mathcal{A}(\tau)}{d \tau}=\frac{\Delta I}{2 e} \exp [-\Gamma \tau / 2]\left[\cos \tilde{\Omega} \tau+\frac{\Gamma}{2 \tilde{\Omega}} \sin \tilde{\Omega} \tau\right],
$$

where $\tilde{\Omega}=\sqrt{\Omega^{2}-\Gamma^{2} / 4}$. Substituting this expression into Eq. (22) we finally obtain

$$
S_{I}(\omega)=S_{0} \operatorname{coth} \frac{e V}{2 T}+\frac{\Omega^{2}(\Delta I)^{2} \Gamma}{\left(\omega^{2}-\Omega^{2}\right)^{2}+\Gamma^{2} \omega^{2}} .
$$

It is easy to check that at zero temperature this result coincides with the results of Refs.10 13. Notice, however, that it does not assume weakly responding detector $(|\Delta I| \ll I)$ as in Refs.10 12. On the other hand, the derivation of Eq. (32) assumes low-transparency QPC as a detector, while a much brgader class of linear detectors was considered in Refs.10 12 .

With the temperature $T$ increase the noise pedestal $S_{0} \operatorname{coth}(\mathrm{eV} / 2 \mathrm{~T})$ increases while the spectral peak around $\Omega$ becomes lower and wider [Fig. 3(a)] because of $\Gamma$ increase [see Eq. (12)]. The integral over the peak,

$$
\int_{0}^{\infty}\left[S_{I}(\omega)-S_{0} \operatorname{coth}(e V / 2 T)\right] \frac{d \omega}{2 \pi}=\frac{(\Delta I)^{2}}{4},
$$

does not depend on the temperature. (As will be seen later, this formula remains valid for $\varepsilon \neq 0$ as well.)

The peak-to-pedestal ("signal-to-noise") ratio

$$
\begin{array}{r}
\frac{S_{I}(\Omega)-S_{0} \operatorname{coth}(e V / 2 T)}{S_{0} \operatorname{coth}(e V / 2 T)}=\frac{(\Delta I)^{2}}{\Gamma S_{0} \operatorname{coth}(e V / 2 T)} \\
=\frac{4}{[\operatorname{coth}(e V / 2 T)]^{2}} \frac{\left(\sqrt{I_{1}}+\sqrt{I_{2}}\right)^{2}}{2\left(I_{1}+I_{2}\right)}
\end{array}
$$

has an upper bound equal to 4 and decreases with temperature as well as due to finite response ratio $|\Delta I| / I_{0}$. In particular, in the case of very strong response, $|\Delta I|=2 I_{0}$ $\left(I_{2}=0\right)$, the upper bound for the peak-to-pedestal ratio is 2 instead of 4 [see Fig. 3(b)]. Overall, the effect of the finite response on the spectral peak shape is similar to the effect of detector nonideality. 10

Let us emphasize that the derivation of Eq. (32) did not use any assumption about the magnitude of the coupling $\mathcal{C} \equiv \hbar(\Delta I)^{2} / S_{0} H$ between the qubit and detector, so Eq. (32) remains valid even when the oscillations are destroyed due to strong coupling ("quantum Zeno" effect) and replaced by a telegraph noise. The analysis of the finite coupling effect is completely similar to that of Refs 19.11. In particular, the quality factor of oscillations (disregarding the noise pedestal) is equal to

$$
\mathcal{Q}=\frac{\Omega}{\Gamma}=\frac{8}{\mathcal{C}} \frac{\left(\sqrt{I_{1}}+\sqrt{I_{2}}\right)^{2}}{4 I_{0}} \frac{1}{\operatorname{coth}(e V / 2 T)}
$$

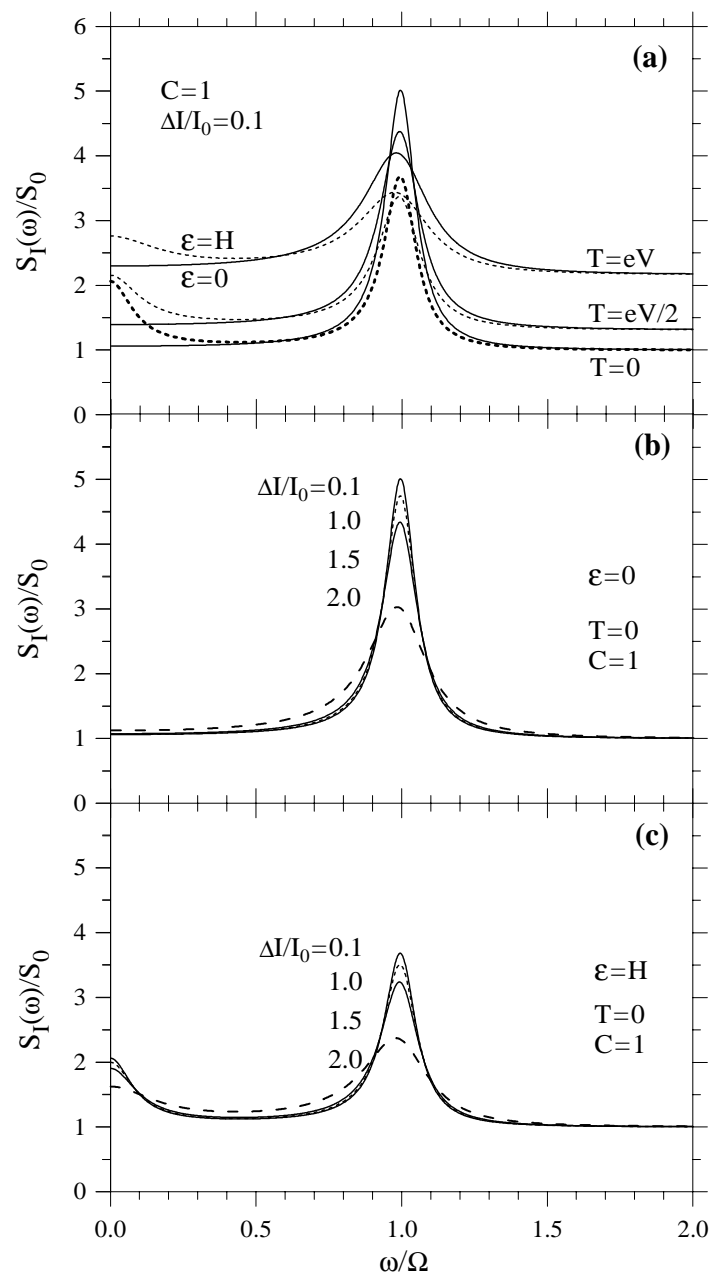

FIG. 3. (a): Spectral density $S_{I}(\omega)$ of the detector current in a weakly coupled $(\mathcal{C}=1)$ and weakly responding $\left(\Delta I / I_{0}=0.1\right)$ regime at detector temperatures $T=0, \mathrm{eV} / 2$, and $e V$ for a symmetric, $\varepsilon=0$ (solid lines), and asymmetric qubit with $\varepsilon=H$ (dotted lines). (b): $S_{I}(\omega)$ for a symmetric qubit and weakly coupled detector $(\mathcal{C}=1)$ at $T=0$ for several response ratios $\Delta I / I_{0}=0.1,1,1.5$, and 2. (c): the same as in (b) for an asymmetric qubit with $\varepsilon=H$.

and the transition into overdamped regime occurs at $\mathcal{Q}<$ $1 / 2$.

For an asymmetric qubit, $\varepsilon \neq 0$, the analytical solution of Eqs. (28)-(30) is too lengthy, so it is easier to use numerical calculations and then calculate the Fourier transform (22) also numerically [Figs. $3(\mathrm{a})$ and $3(\mathrm{c})$ ]. In the next Section we will show that the result is still equivalent to the results of Refs. 1013 (within the common validity range). 


\section{EQUIVALENCE TO THE MASTER EQUATION APPROACH}

Let us remind that the master equation approach $12, \mathrm{n} 1$ assumes no correlation between the detector noise and qubit evolution and treats the oscillating part of the current $I(t)$ as being proportional to the quantum operator $\hat{z}(t)$, so the spectral density $S_{I}(\omega)$ should be calculated as

$$
S_{I}(\omega)=S_{\text {det }}+\frac{(\Delta I)^{2}}{4} 4 \int_{0}^{\infty} K_{\hat{z}}(\tau) \cos (\omega \tau) d \tau
$$

where $S_{\text {det }}$ is the detector noise and $K_{\hat{z}}(\tau) \equiv\langle\hat{z}(t+$ $\tau) \hat{z}(t)\rangle$ is the correlation function of $\hat{z}$. In the case of a weakly responding detector the detector noise level does not depend on the qubit state and $S_{d e t}=$ $S_{0} \operatorname{coth}(\mathrm{eV} / 2 \mathrm{~T})$. The same formula remains valid in the case of moderate or strong response because at high frequency $S_{I}(\infty)=2 e\left(\left\langle I^{+}\right\rangle+\left\langle I^{-}\right\rangle=2 e\langle I\rangle \operatorname{coth}(e V / 2 T)\right.$ and the average current $\langle I\rangle$ remains to be equal to $I_{0}$. As shown in Refs.10.11, the correlation function $K_{\hat{z}}$ is equal to the value of $z(\tau)=\rho_{11}(\tau)-\rho_{22}(\tau)$ obtained from the master equation (10)-(11) with initial condition $\rho_{11}(0)=1, \rho_{22}(0)=\rho_{12}(0)=0$. (Actually, in those papers only the case of a weakly responding detector at zero temperature has been considered; however, the method can be easily generalized, since formally the only change in the master equation is a different $\Gamma$.)

Let us show that $S_{I}(\omega)$ calculated in this way coincides with the result obtained from Eq. (22) at arbitrary qubit asymmetry $\varepsilon$. For this purpose we introduce the new variable $a \equiv(2 e / \Delta I) \dot{\mathcal{A}}$ [so that we need to prove $a(\tau)=$ $\left.K_{\hat{z}}(\tau)\right]$ and from Eqs. (28)-(30) derive a new system of equations

$$
\begin{aligned}
& \dot{a}=-4(H / \hbar) y, \\
& \dot{y}=(\varepsilon / \hbar) x+(H / \hbar) a-\Gamma y, \\
& \dot{x}=-(\varepsilon / \hbar) y-\Gamma x,
\end{aligned}
$$

where $y \equiv(2 e / \Delta I) \dot{\mathcal{Y}}$ and $x \equiv(2 e / \Delta I) \dot{\mathcal{X}}$. It is easy to see that these equations coincide with the master equations (10)-(11) for $z, \operatorname{Im} \rho_{12}$, and $\operatorname{Re} \rho_{12}$, respectively. Since $\mathcal{A}(0)=\mathcal{X}(0)=\mathcal{Y}(0)=0$, the initial conditions for new variables are $a(0)=1$ and $x(0)=y(0)=0$, i.e. exactly the initial conditions for $K_{\hat{z}}$ calculation. Therefore $a(\tau)=K_{\hat{z}}(\tau)$ and the spectral density $S_{I}(\omega)$ calculated using the Bloch equations coincides with the result of the master equation approach.

Hence, the analysis of $S_{I}(\omega)$ at finite qubit asymmetry $\varepsilon$ is completely similar to that of Refs.10 12. In particular, finite $\varepsilon$ leads to a decrease of the spectral peak around frequency $\Omega$ and origination of an extra peak around zero frequency (Fig. 3), while the integral (33) does not change [this is a consequence of $\left.K_{\hat{z}}(0)=1\right]$. An analytical expression

$$
S_{I}(\omega)=S_{0} \operatorname{coth} \frac{e V}{2 T}+\frac{\varepsilon^{2}(\Delta I)^{2} /\left(4 H^{2} \Gamma\right)}{1+\left(\omega \hbar^{2} \Omega^{2} / 4 H^{2} \Gamma\right)^{2}}
$$

$$
+\frac{(\Delta I)^{2} /\left[\Gamma\left(1+\varepsilon^{2} / 2 H^{2}\right)\right]}{1+\left[(\omega-\Omega) 2 /\left[\Gamma\left(1+\varepsilon^{2} / \hbar^{2} \Omega^{2}\right)\right]\right]^{2}}
$$

can be obtained in the limit $\Gamma \ll \Omega$.

Notice that for both Bloch and master equation approaches the case of a finite detector response does not formally differ from the case of a weakly responding detector (only the value of the ensemble decoherence rate $\Gamma$ changes). For the Bayesian approach these two cases are significantly different, so the generalization of the result 10 for $S_{I}(\omega)$ to a finite detector response (considered in the next Section) is not trivial.

\section{GENERALIZATION OF THE BAYESIAN RESULTS FOR $S_{I}(\omega)$}

The Bayesian results for $S_{I}(\omega)$ have been derived in Ref.10 only for the case of a weakly responding detector, in which the detector current $I(t)$ can be considered as continuoys and the qubit evolution is described by the equation 19 , 9

$$
\begin{aligned}
\dot{\rho}_{11}= & -\dot{\rho}_{22}=\rho_{11} \rho_{22} \frac{2 \Delta I}{S_{0}}\left[I(t)-I_{0}\right]-2 \frac{H}{\hbar} \operatorname{Im} \rho_{12}, \\
\dot{\rho}_{12}= & -\left(\rho_{11}-\rho_{22}\right) \frac{\Delta I}{S_{0}}\left[I(t)-I_{0}\right] \rho_{12}-\gamma \rho_{12} \\
& +\mathrm{i} \frac{\varepsilon}{\hbar} \rho_{12}+\mathrm{i} \frac{H}{\hbar}\left(\rho_{11}-\rho_{22}\right),
\end{aligned}
$$

where $\gamma=\Gamma-(\Delta I)^{2} / 4 S_{0}$ is the qubit decoherence rate without ensemble averaging [for our model $\gamma / \Gamma=$ $\cosh ^{-2}(e V / 2 T)$, so that $\gamma=0$ at $\left.T=0\right]$ and the statistics of $I(t)$ can be modeled as

$$
I(t)-I_{0}=\left[\rho_{11}(t)-\rho_{22}(t)\right] \Delta I / 2+\xi(t),
$$

where $\xi(t)$ is the white noise with the spectral density $S_{\xi}=S_{0}$. Notice the significant difference in the meaning of $\rho_{i j}$ in the Bayesian equations and in the master equation since Eqs. (41)-(42) describe individual qubit evolution without ensemble averaging. Also notice that the detector noise $\xi(t)$ is now significantly correlated with the qubit evolution $\rho_{i j}(t)$.

To consider the case of a detector with finite response factor $|\Delta I| / I_{0}$, we necessarily need to take into account individual tunnel events in the detector because $\Gamma$ becomes comparable to $I_{0} / e$. Hence, the current is not continuous any more and we have to use generalized Bayesian equations (which are essentially similar to the equations of the quantum jumps formalism 22). Then the qubit evolution during the time intervals between tunnel events in the detector is continuous and given by the small-time Bloch equations for $\rho_{i j}^{0}$ with the restored normalization:

$\dot{\rho}_{11}=-\dot{\rho}_{22}=-\frac{\Delta I}{e} \operatorname{coth}\left(\frac{e V}{2 T}\right) \rho_{11} \rho_{22}-2 \frac{H}{\hbar} \operatorname{Im} \rho_{12}$, 


$$
\begin{aligned}
\dot{\rho}_{12}= & \frac{\Delta I}{2 e} \operatorname{coth}\left(\frac{e V}{2 T}\right)\left(\rho_{11}-\rho_{22}\right) \rho_{12} \\
& +\mathrm{i} \frac{\varepsilon}{\hbar} \rho_{12}+\mathrm{i} \frac{H}{\hbar}\left(\rho_{11}-\rho_{22}\right),
\end{aligned}
$$

while each tunnel event in the detector (at time $t=t_{k}$ ) causes abrupt change (collapse) of the qubit state:

$$
\begin{aligned}
& \rho_{11}\left(t_{k}+0\right)=\frac{I_{1} \rho_{11}\left(t_{k}-0\right)}{I_{1} \rho_{11}\left(t_{k}-0\right)+I_{2} \rho_{22}\left(t_{k}-0\right)}, \\
& \rho_{22}\left(t_{k}+0\right)=1-\rho_{11}\left(t_{k}+0\right), \\
& \frac{\rho_{12}\left(t_{k}+0\right)}{\rho_{12}\left(t_{k}-0\right)}=\left[\frac{\rho_{11}\left(t_{k}+0\right) \rho_{22}\left(t_{k}+0\right)}{\rho_{11}\left(t_{k}-0\right) \rho_{22}\left(t_{k}-0\right)}\right]^{1 / 2} .
\end{aligned}
$$

[Actually, in Eq. (46) instead of $I_{i}$ it is better to write $I_{i}^{+}$ if the tunneling is in the positive direction and $I_{i}^{-}$if it is in the negative direction; however, the corresponding temperature factors cancel each other.] It is interesting to note that the generalized Bayesian equations do not contain any decoherence term even at finite temperature. This is because our model of the low-transparency QPC describes an ideal detectorl ${ }^{9}$ and counting tunnel events in both directions gives more information than measurement of only total current $\left(I^{+}-I^{-}\right)$assumed in Eqs. (41) $-(43)$.

For the evolution simulation Eqs. (44)-(48) should be complemented by the statistics of tunnel events in the detector. This statistics is described by the (varying) rates $p^{+}$and $p^{-}$of tunneling events in the positive and negative directions, respectively:

$$
\begin{aligned}
& p^{+}(t)=\left(I_{1}^{+} / e\right) \rho_{11}(t)+\left(I_{2}^{+} / e\right) \rho_{22}(t) \\
& p^{-}(t)=\left(I_{1}^{-} / e\right) \rho_{11}(t)+\left(I_{2}^{-} / e\right) \rho_{22}(t) .
\end{aligned}
$$

It is important to notice that ensemble averaging of evolution equations (44)-48) over random moment of tunneling events described by Eqs. (49)-(50) leads 9 to the conventional master equation (10)-(11) with the ensemble decoherence rate $\Gamma$ given by Eq. (12).

Tecalculate $S_{I}(\omega)$ we will use the method developed in Ref.2 9 and write the current correlation function $K_{I}(\tau) \equiv$ $\langle I(t+\tau) I(t)\rangle=K_{I}(-\tau)$ at $\tau \geq 0$ as

$$
\begin{aligned}
K_{I}(\tau)= & s \delta(\tau)+\left\langle I^{+}\right\rangle e\left[p^{+}(\tau \mid+)-p^{-}(\tau \mid+)\right] \\
& -\left\langle I^{-}\right\rangle e\left[p^{+}(\tau \mid-)-p^{-}(\tau \mid-)\right],
\end{aligned}
$$

where $s=S_{I}(\infty) / 2$ determines the pedestal of $S_{I}(\omega)$ and $p^{ \pm}(\tau \mid \pm)$ is the average rate of tunneling in the positive $\left(p^{+}\right)$or negative $\left(p^{-}\right)$direction at time $t+\tau$, for the condition that at time $t$ a tunneling in the positive $(\mid+)$ or negative $(\mid-)$ direction has occurred.

The value of $s$ should be chosen in a way to provide the correct value of $S_{I}(\infty)=S_{0} \operatorname{coth}(e V / 2 T)$ which can be calculated in the same manner as in the previous Section. For the calculation of $p^{ \pm}(\tau \mid+)$ let us notice that as seen from Eq. (46), after the positive tunneling (at $\tau=0$ ) the value of $z=\rho_{11}-\rho_{22}$ is equal to

$$
z(t+0 \mid+)=\frac{I_{1} \rho_{11}-I_{2} \rho_{22}}{I_{1} \rho_{11}+I_{2} \rho_{22}},
$$

where $\rho_{i i}$ are taken before the tunneling. Averaging $z(t+0 \mid+)$ over the positive tunneling events [or, equivalently, over time with the weight factor $p^{+}(t)$, which is proportional to the denominator of Eq. (52)], we get $\langle z(t+0 \mid+)\rangle=\left\langle I_{1} \rho_{11}-I_{2} \rho_{22}\right\rangle /\langle I\rangle$, expressed via simple averaging over time. Since $\left\langle\rho_{11}\right\rangle=\left\langle\rho_{22}\right\rangle=1 / 2$, the expression can be further simplified: $\langle z(t+0 \mid+)\rangle=$ $\Delta I / 2\langle I\rangle$. Similar calculation shows that $\left\langle\rho_{12}(t+0 \mid+)\right\rangle=$ $\left\langle\rho_{12}\right\rangle\left(I_{1} I_{2}\right)^{1 / 2} /\langle I\rangle=0$.

It is sufficient to know $\langle z(t+0 \mid+)\rangle$ and $\left\langle\rho_{12}(t+0 \mid+)\right\rangle$ to calculate $p^{ \pm}(\tau \mid+)$ because of the linearity of the averaged evolution equations (10)-(11) in terms of $z$ and $\rho_{12}$ and linearity of Eqs. (49)-(50). Using Eqs. (10)-(11) with averaged initial conditions at $\tau=0$, we can show

$$
p^{ \pm}(\tau \mid+)-\frac{\left\langle I^{ \pm}\right\rangle}{e}=\frac{I_{1}^{ \pm}-I_{2}^{ \pm}}{2 e} \frac{\Delta I}{2\langle I\rangle} z(\tau),
$$

where $z(\tau)$ is calculated from Eqs. (10)-(11) starting from initial condition $\rho_{11}=1, \rho_{22}=\rho_{12}=0$. Finally noticing that the expressions do not depend on the direction of tunneling at $\tau=0$ and combining the terms in Eq. (51) we obtain

$$
K_{I}(\tau>0)=\langle I\rangle^{2}+\frac{(\Delta I)^{2}}{4} z(\tau)
$$

with the same $z(\tau)$ as above. The constant term $\langle I\rangle^{2}$ does not contribute to the Fourier transform $S_{I}(\omega)=$ $2 \int_{-\infty}^{\infty} K_{I}(\tau) \cos (\omega \tau) d \tau$ (formally it leads to a $\delta$-function at $\omega=0$ ), while the second term of Eq. (54) gives the same contribution as the second term of Eq. (36). Consequently, the calculation of $S_{I}(\omega)$ using the generalized Bayesian approach leads to the same result as the master equation approach.

\section{CONCLUSION}

The main result of this paper is the development of a method of $S_{I}(\omega)$ calculation for a detector measuring quantum coherent oscillations of a qubit, based on the microscopic Bloch equations, 26 which couple the qubit and detector degrees of freedom. As the detector we assumed a low-transparency QPC (tunnel junction). We have shown that $S_{I}(\omega)$ calculated in this way formally coincides with the results obtained previously 1013 by the Bayesian, master equation, and quantum jumps methods, though in this paper we have considered a wider validity range. In particular, our formalism takes into account finite detector temperature $T$ and assumes arbitrary detector response $|\Delta I| / I_{0}$.

Besides that, we have generalized the Bayesian method of $S_{I}(\omega)$ calculation to the case of arbitrary detector response and temperature. (The generalization of the master equation method is formally trivial.) We have proven 
that the results of all three methods still coincide in such generalized case.

The model we have considered describes essentially an ideal detector. The detector nonideality can be phenomenologically taken into account by introducing an extra dephasing term into the Bloch, master, and Bayesian equations, containing $\dot{\rho}_{12}$. This will lead to an increase of the ensemble decoherence rate $\Gamma$ and, therefore, to a wider and lower peak of $S_{I}(\omega)$, corresponding to the qubit oscillations. 1013 Similar procedure can be done to take into account a qupit-controlled change of the detector tunneling phase 1113 [then $\Gamma=$ $\left(2 I_{0}-\sqrt{4 I_{0}^{2}-(\Delta I / \cos \theta)^{2}}\right)(2 e)^{-1} \operatorname{coth}(e V / 2 T)$ where $\left.\theta=\arg \left(M^{*} \Delta M\right)\right]$ even thoygh detector is still ideal in the generalized sense.9.12,13 The effect of a weak extra coupling between the qubit and a passive finitetemperature environment (with temperature different from $T$-can be taken into account in a way similar to Refs.10 12. We did not consider these effects in the present paper because their treatment is exactly the same as in previous papers.

An experimental measurement of $S_{I}(\omega)$ and verification of the upper bound $\left(\leq 4 S_{0}\right)$ for the spectral peak corresponding to qubit oscillations seems to be the easiest experiment related to a continuous monitoring of a nontrivial single qubit evolution. This makes it preferable for sooner realization in comparison with mere interesting but more difficult proposed experiment 19.20 .30 on monitoring and continuous collapse of a solid-state qubit.

The authors thank L. Fedichkin for useful discussions. The work was supported by NSA and ARDA under ARO grant DAAD19-01-1-0491.

${ }^{1}$ L. D. Landau and E. M. Lifshitz, Quantum mechanics: nonrelativistic theory (Oxford, NY, 1977).

${ }^{2}$ C. Bennett, Phys. Today 48 (10), 24 (1995).

${ }^{3}$ Y. Nakamura, Yu. A. Pashkin, and J. S. Tsai, Nature (London) 398, 786 (1999); Phys. Rev. Lett. 87, 246601 (2001).

${ }^{4}$ C. H. van der Wal, A. C. J. ter Haar, F. K. Wilhelm, R. N. Schouten, C. J. P. M. Harmans, T. P. Orlando, S. Lloyd, and J. E. Mooij, Science 290, 773 (2000).

${ }^{5}$ J. R. Friedman, V. Patel, W. Chen, S. K. Tolpygo, and J. E. Lukens, Nature (London) 406, 43 (2000).

${ }^{6}$ T. H. Stievater, X. Li, D. G. Steel, D. Gammon, D. S. Katzer, D. Park, C. Piermarocchi, and L. J. Sham, Phys. Rev. Lett. 87, 133603 (2001); H. Htoon, T. Takagahara, D. Kulik, O. Baklenov, A. L. Holmes, and C. K. Shih, Phys. Rev. Lett. 88, 087401 (2002).

${ }^{7}$ E. Buks, R. Schuster, M. Heiblum, D. Mahalu, and V. Umansky, Nature (London) 391, 871 (1998).

${ }^{8}$ D. Sprinzak, E. Buks, M. Heiblum, and H. Shtrikman, Phys. Rev. Lett. 84, 5820 (2000).

${ }^{9}$ A. N. Korotkov, Phys. Rev. B 63, 115403 (2001).
${ }^{10}$ A. N. Korotkov, Phys. Rev. B 63, 085312 (2001).

11 A. N. Korotkov and D. V. Averin, Phys. Rev. B 64, 165310 (2001).

${ }^{12}$ D. V. Averin, cond-mat/0004364.

${ }^{13}$ H. S. Goan and G. J. Milburn, Phys. Rev. B 64, 235307 (2001).

14 M.-S. Choi, F. Plastina, and R. Fazio, Phys. Rev. Lett. 87, 116601 (2001).

${ }^{15}$ D. V. Averin, cond-mat/0202082.

${ }^{16}$ D. Mozyrsky, L. Fedichkin, S. A. Gurvitz, and G. P. Berman, cond-mat/0201325.

${ }^{17}$ G. Hackenbroich, B. Rosenow, and H. A. Weidenmüller, Phys. Rev. Lett. 81, 5896 (1998).

${ }^{18}$ Y. Makhlin, G. Schön, and A. Shnirman, Phys. Rev. Lett. 85, 4578 (2000).

19 A. N. Korotkov, Phys. Rev. B 60, 5737 (1999).

${ }^{20}$ R. Ruskov and A. N. Korotkov, cond-mat/0107280; in Quantum confinement, ed. by M. Cahay et al. (Electrochemical Society, 2001), p. 287.

${ }^{21}$ H. M. Wiseman and G. J. Milburn, Phys. Rev. A 47, 1652 (1993).

${ }^{22}$ H.-S. Goan, G. J. Milburn, H. M. Wiseman, and H. B. Sun, Phys. Rev. B 63, 125326.

${ }^{23}$ Y. Manassen, R. J. Hamers, J. E. Demuth, and A. J. Castellano, Phys. Rev. Lett. 62, 2531 (1989); Y. Manassen, I. Mukhopadhyay, and N. R. Rao, Phys. Rev. B 61, 16223 (2000).

${ }^{24}$ C. Durkan and M. E. Welland, Appl. Phys. Lett. 80, 458 (2002).

${ }^{25}$ C. Durkan, private communication.

${ }^{26}$ S. A. Gurvitz, Phys. Rev. B 56, 15215 (1997).

${ }^{27}$ In particular, we should always assume $\mathrm{eV} \gg \hbar \Omega$. To consider individual electron tunneling in the detector, we also need $e V / \hbar \gg I / e$ that translates into a condition of sufficiently large resistance: $R \gg \hbar / e^{2}$ (even for a multimode QPC). However, in the weakly responding regime the second condition can be relaxed to $\mathrm{eV} / \hbar \gg(\Delta I)^{2} / S_{0}$ that translates into $R \gg\left(\Delta I / I_{0}\right)^{2} \hbar / e^{2}$ (the transparency of each mode is still small).

${ }^{28}$ D. K. C. MacDonald, Noise and Fluctuations: an Introduction (Wiley, New York, 1962), Ch. 2.2.1.

${ }^{29}$ A. N. Korotkov, Phys. Rev. B 49, 10381 (1994).

${ }^{30}$ A. N. Korotkov, Phys. Rev. B 64, 193407 (2001). 